\title{
Two Condensed Macro-Elements with Full Approximation Power
}

\author{
Peter Alfeld ${ }^{1)}$, Larry L. Schumaker ${ }^{2)}$, and Tatyana Sorokina ${ }^{3)}$
}

\begin{abstract}
A variant of the classical $C^{1}$ Powell-Sabin-12 macro-element is defined that has the same approximation power, but has fewer degrees of freedom and only requires vertex data. The same idea is then applied to a related $C^{1}$ trivariate macro-element.
\end{abstract}

\section{$\S 1$. Introduction}

Suppose $\triangle$ is a triangulation of a domain $\Omega \subset \mathbb{R}^{2}$ or a tetrahedral partition of a domain in $\mathbb{R}^{3}$, and that $\triangle_{R}$ is a refinement of $\triangle$ obtained by applying a given splitting procedure to each triangle or tetrahedron in $\triangle$. Suppose in addition that $\mathcal{S}\left(\triangle_{R}\right)$ is a polynomial spline space defined on $\triangle_{R}$ such that every spline $s \in \mathcal{S}\left(\triangle_{R}\right)$ is uniquely determined by the values of $s$ and its gradients at some collection of points in $\Omega$. Then (cf. [4]) $\mathcal{S}\left(\triangle_{R}\right)$ is called a macro-element space provided that for each triangle (tetrahedron) in $\triangle,\left.s\right|_{T}$ is uniquely determined by the data at points in $T$.

There are many examples of both bivariate and trivariate macro-element spaces. For complete details, see [4]. Macro-element spaces are particularly important in applications since they generally have stable local bases and have full approximation power. This means that they approximate sufficiently smooth functions to $\mathcal{O}\left(|\triangle|^{d+1}\right)$, where $d$ is the degree of the splines, and $|\triangle|$ is the mesh size of $\triangle$, i.e., the length of the longest edge in $\triangle$.

In applications, and in particular for scattered data fitting and the solution of boundary-value problems by the finite-element method, it is advantageous to work with macro-element spaces that produce a given order of approximation while involving the least number of degrees of freedom. Degrees of freedom can frequently be removed from macro-elements by a process called condensation, but typically at the cost of not preserving full approximation power. The purpose of this paper is to show that for one well-known bivariate macro-element (the classical bivariate

1) Department of Mathematics, University of Utah, 155 South 1400 East, JWB 233, Salt Lake City, Utah 84112-0090, pa@math.utah.edu

2) Department of Mathematics, Vanderbilt University, Nashville, TN 37240, larry.schumaker@vanderbilt.edu.

3) Department of Mathematics, Towson University, Towson, MD 21252, tsorokina@towson. edu 
$C^{1}$ Powell-Sabin-12 macro-element) and a related trivariate one described recently in [8], it is possible to condense without losing any approximation power.

Our condensed macro-element spaces will be constructed by requiring that certain cross boundary derivatives be polynomials instead of piecewise polynomials. For the $C^{1}$ Powell-Sabin-12 element this was suggested already in [6], although there is no discussion of approximation power there. In the trivariate case, this approach was used to condense the second element in [2].

After some preliminaries in Section 2, we describe in Section 3 how to condense the bivariate Powell-Sabin 12 element. In Section 4 we apply the same technique to the tetrahedral quadratic element described in [8]. This scheme is related to the bivariate scheme since it makes use of the PS-12 split on each tetrahedral face. In Section 5 we demonstrate numerically that the condensation has only a small effect on the error in the interpolant. We close the paper with some remarks.

\section{$\S 2$. Preliminaries}

Throughout this paper we follow the notation of the book [4]. Let $\triangle$ be a triangulation or tetrahedral partition of a domain $\Omega$ in $\mathbb{R}^{2}$ or $\mathbb{R}^{3}$. Given integers $0 \leq r<d$, we write

$$
\mathcal{S}_{d}^{r}(\triangle):=\left\{s \in C^{r}(\Omega):\left.s\right|_{T} \in \mathcal{P}_{d} \text { all } T \in \triangle\right\},
$$

where $\mathcal{P}_{d}$ is the space of polynomials of degree $d$. Since we will work with polynomials in 1,2 , and 3 variables in the sequel, from now on we write $\mathcal{P}_{d}^{\mu}$ for the space of polynomials in $\mu$ variables.

Suppose $\mathcal{N}:=\left\{\lambda_{i}\right\}_{i=1}^{n}$ is a set of linear functionals of the form $\varepsilon_{\eta_{i}} D_{x}^{\alpha_{i}} D_{y}^{\beta_{i}}$, where $\varepsilon_{\eta}$ denotes point evaluation at the point $\eta$. Let $\mathcal{S}(\triangle)$ be a subspace of $\mathcal{S}_{d}^{r}(\triangle)$. Then $\mathcal{N}$ is called a nodal determining set for $\mathcal{S}(\triangle)$ provided that if $s \in \mathcal{S}(\triangle)$ and $\lambda_{i} s=0$ for all $i=1, \ldots, n$, then $s \equiv 0$. A smallest set with this property is called a nodal minimal determining set (NMDS). If $\mathcal{N}$ is a NMDS, then for every sufficiently smooth function $f$, there exists a unique spline $s \in \mathcal{S}(\triangle)$ satisfying the Hermite interpolation conditions

$$
\lambda_{i} s=\lambda_{i} f, \quad i=1, \ldots, n,
$$

and the cardinality of $\mathcal{N}$ equals the dimension of $\mathcal{S}(\triangle)$.

A NMDS $\mathcal{N}$ is called local and stable provided there exists an integer $\ell$ and a constant $K$ depending on the smallest angle in the partition such that

$$
\left|c_{\xi}\right| \leq K \sum_{i=1}^{\bar{m}}|T|^{i}|s|_{i, \Omega_{T}},
$$

for all B-coefficients $c_{\xi}$ associated with domain points $\xi$ lying in $T$. Here $|T|$ denotes the area (volume in the trivariate case) of $T, \Omega_{T}:=\operatorname{star}^{\ell}(T)$, and $\bar{m}$ is the maximum derivative needed to define $\mathcal{N}$. As usual, $|s|_{i, \Omega_{T}}$ denotes the standard Sobolev seminorm, and $\operatorname{star}^{\ell}(T)$ is the $\ell$-th order star of $T$ defined recursively by $\operatorname{star}^{0}(T)=T$, and $\operatorname{star}^{\ell}(T)=\bigcup\left\{t \in \triangle: t \cap \operatorname{star}^{\ell-1}(T) \neq \emptyset\right\}$. Given a stable local NMDS for a spline space $\mathcal{S}(\triangle)$, there is a simple way to construct a corresponding stable local basis, see pp. 144 and 491 of [4]. 


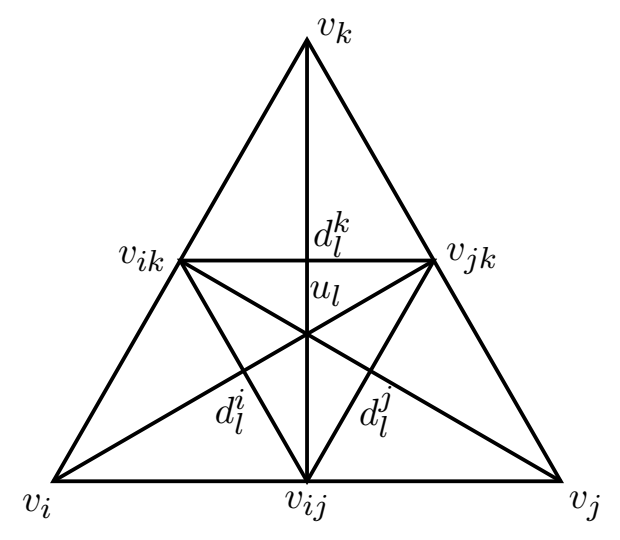

Fig. 1. Vertices of the PS-12 split.
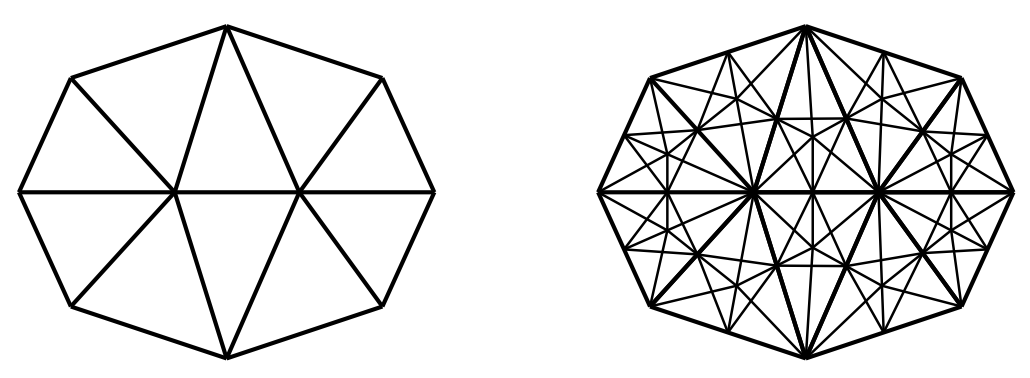

Fig. 2. A triangulation and its Powell-Sabin-12 refinement.

\section{§3. A Condensed Powell-Sabin-12 Macro-Element Space}

Given a triangulation $\triangle$ of $\Omega \subset \mathbb{R}^{2}$, let $\mathcal{V}, \mathcal{E}$ be the sets of vertices and edges, respectively. We write $\triangle_{P S 12}$ for the refined triangulation obtained by splitting each triangle $T$ in $\triangle$ into twelve subtriangles using the barycenters and midpoints of edges as shown in Figs. 1 and 2 . The bivariate spline space $\mathcal{S}_{2}^{1}\left(\triangle_{P S 12}\right)$ is called the Powell-Sabin-12 (PS-12) macro-element space, first introduced in [6]. It is well known (cf. Theorem 6.13 in [4]) that the dimension of $\mathcal{S}_{2}^{1}\left(\triangle_{P S 12}\right)$ is $3 n_{V}+n_{E}$.

It is easy to describe a NMDS for $\mathcal{S}_{2}^{1}\left(\triangle_{P S 12}\right)$. For each vertex $v$ of $\triangle$, let $\mathcal{N}_{v}:=\left\{\varepsilon_{v}, \varepsilon_{v} D_{x}, \varepsilon_{v} D_{y}\right\}$, where, as before, $\varepsilon_{v}$ denotes point-evaluation at $v$. For each edge $e$ of $\triangle$, let $e_{m}$ be the midpoint of $e$, and let $D_{n_{e}}$ be the derivative in a unit direction $n_{e}$ perpendicular to $e$, and let $\mathcal{N}_{e}:=\left\{\varepsilon_{e_{m}} D_{n_{e}}\right\}$. Then (cf. Theorem 6.15 of $[4])$,

$$
\mathcal{N}=\bigcup_{v \in \mathcal{V}} \mathcal{N}_{v} \cup \bigcup_{e \in \mathcal{E}} \mathcal{N}_{e},
$$

is a stable local NMDS for $\mathcal{S}_{2}^{1}\left(\triangle_{P S 12}\right)$. Moreover, the corresponding dual $\mathcal{N}$-basis for $\mathcal{S}_{2}^{1}\left(\triangle_{P S 12}\right)$ is a stable local basis. It follows from Theorem 5.26 of [4] that $\mathcal{S}_{2}^{1}\left(\triangle_{P S 12}\right)$ approximates smooth functions to optimal order $\mathcal{O}\left(|\triangle|^{3}\right)$.

We now define a subspace of $\mathcal{S}_{2}^{1}\left(\triangle_{P S 12}\right)$ which also is a macro-element space with full approximation power, but which requires only nodal data at the vertices of $\triangle$. It is clear that for every $s \in \mathcal{S}_{2}^{1}\left(\triangle_{P S 12}\right)$ and every edge $e$ of $\triangle, D_{n_{e}} s$ is a 
piecewise linear function on $e$. We define our condensed macro-element space by requiring that these cross derivatives be linear rather than piecewise linear.

Definition 3.1. Given $\triangle_{P S 12}$, we define the associated condensed $C^{1}$ quadratic PS-12 macro-element space to be

$$
\widetilde{\mathcal{S}}_{2}^{1}\left(\triangle_{P S 12}\right):=\left\{s \in \mathcal{S}_{2}^{1}\left(\triangle_{P S 12}\right):\left.D_{n_{e}} s\right|_{e} \in \mathcal{P}_{1}^{1} \text { for each edge } e \text { of } \triangle\right\}
$$

Theorem 3.2. The dimension of $\widetilde{\mathcal{S}}_{2}^{1}\left(\triangle_{P S 12}\right)$ is $3 n_{V}$, and $\widetilde{\mathcal{N}}:=\bigcup_{v \in \mathcal{V}} \mathcal{N}_{v}$ is a stable local NMDS.

Proof: By the definition of $\widetilde{\mathcal{S}}_{2}^{1}\left(\triangle_{P S 12}\right)$, if we set the values and gradients of $s \in \widetilde{\mathcal{S}}_{2}^{1}\left(\triangle_{P S 12}\right)$ at the vertices of $\triangle$, then the cross derivatives $D_{n_{e}}$ at the midpoints of the edges are uniquely determined by requiring $\left.D_{n_{e}} s\right|_{e}$ to be linear, see also the explicit formulae (3.2) provided at the end of this section. The result then follows from the fact that $\mathcal{N}$ is a stable local NMDS for $\mathcal{S}_{2}^{1}\left(\triangle_{P S 12}\right)$.

Corollary 3.3. For each $f \in C^{1}(\Omega)$, there exists a unique $s \in \widetilde{\mathcal{S}}_{2}^{1}\left(\triangle_{P S 12}\right)$ such that

$$
s(v)=f(v) \quad \text { and } \quad \nabla s(v)=\nabla f(v), \quad \text { for all } v \in \mathcal{V} .
$$

Moreover if $f \in \mathcal{P}_{2}^{2}$ then $s \equiv f$.

Proof: The first statement is obvious due to the definition of $\widetilde{\mathcal{N}}$. Now suppose $f \in \mathcal{P}_{2}^{2}$. Then for each edge $e$ of $\triangle,\left.D_{n_{e}} s\right|_{e} \in \mathcal{P}_{1}^{1}$. Now since $\mathcal{P}_{2}^{2} \subset \widetilde{\mathcal{S}}_{2}^{1}\left(\triangle_{P S 12}\right)$ and there is a unique $s \in \widetilde{\mathcal{S}}_{2}^{1}\left(\triangle_{P S 12}\right)$ satisfying (3.1), it follows that $s \equiv f$.

Since $\widetilde{\mathcal{N}}$ is stable and local, it follows from Theorem 5.26 of [4] that the Hermite interpolating spline of Corollary 3.3 approximates smooth functions to optimal order.

For convenience in writing a program to compute the spline $s$ satisfying (3.1), we now give explicit formulae for all of its B-coefficients. There is one B-coefficient associated with each vertex of $\triangle_{P S 12}$, and one associated with the midpoint of each edge. If $v$ is a vertex of $\triangle$, we write $c(v)$ for the B-coefficient associated with the domain point $v$, and if $e:=\langle u, v\rangle$ is an edge of $\triangle$, we write $c(u, v)$ for the $\mathrm{B}$-coefficient associated with the domain point at the midpoint of $e$.

For ease in working with the trivariate case in the following section, following the notation used in [8] we write $T:=\left\langle v_{i}, v_{j}, v_{k}\right\rangle$ for a typical macro-triangle, cf., Fig. 1. We write $v_{i j}$ for the midpoint of the edge $e_{i j}:=\left\langle v_{i}, v_{j}\right\rangle$, with similar notation for the other edges of $T$. Let $u_{l}:=\left(v_{i}+v_{j}+v_{k}\right) / 3$ be the barycenter of $T$, and let $d_{l}^{i}:=\left(2 v_{i}+v_{j}+v_{k}\right) / 4$ be the midpoint of the edge $\left\langle v_{i j}, v_{i k}\right\rangle$, with similar definitions for $d_{l}^{j}$ and $d_{l}^{k}$. Here the sub- and super-script $l$ is extraneous, but it is useful when we deal with the trivariate case in Sect. 4. Then by standard Bernstein 
Bézier arguments, we get the following formulae for the B-coefficients of $s$ :

$$
\begin{aligned}
c\left(v_{i}\right) & =f\left(v_{i}\right), \\
c\left(v_{i}, v_{i j}\right) & =c\left(v_{i}\right)+\nabla f\left(v_{i}\right) \circ\left(v_{i j}-v_{i}\right) / 2, \\
c\left(v_{i j}\right) & =\left[c\left(v_{i}, v_{i j}\right)+c\left(v_{j}, v_{i j}\right)\right] / 2, \\
c\left(v_{i}, d_{l}^{i}\right) & =\left[c\left(v_{i}, v_{i j}\right)+c\left(v_{i}, v_{i k}\right)\right] / 2, \\
c\left(v_{i j}, d_{l}^{i}\right) & =\left[3 c\left(u_{l}, v_{i j}\right)+c\left(v_{i}, v_{i j}\right)\right] / 4, \\
c\left(d_{l}^{i}\right) & =\left[c\left(d_{l}^{i}, v_{i j}\right)+c\left(d_{l}^{i}, v_{i k}\right)\right] / 2, \\
c\left(u_{l}, d_{l}^{i}\right) & =\left[c\left(u_{l}, v_{i j}\right)+c\left(u_{l}, v_{i k}\right)\right] / 2, \\
c\left(u_{l}\right) & =\left[c\left(u_{l}, v_{i j}\right)+c\left(u_{l}, v_{i k}\right)+c\left(u_{l}, v_{j k}\right)\right] / 3, \\
c\left(d_{l}^{i}, u_{l}\right) & =\left[c\left(v_{i j}, u_{l}\right)+c\left(v_{i k}, u_{l}\right)\right] / 2,
\end{aligned}
$$

where it remains to give a formula for $c\left(u_{l}, v_{i j}\right)$ in terms of coefficients in the first three equations of (3.2). Note that each of the formulae in (3.2) is to be applied with permutations of $i, j, k$ to get the other coefficients of $s$.

Suppose $e:=\left\langle v_{i}, v_{j}\right\rangle$, and let $n_{e}$ be the unit vector perpendicular to $e$ and pointing into $T$. To give a formula for $c\left(u_{l}, v_{i j}\right)$, we make use of our requirement that $D_{n_{e}} s$ should be linear along the edge $e$. First, we write

$$
\begin{aligned}
n_{e} & =\alpha_{i}\left(v_{i j}-v_{i}\right)+\beta_{i}\left(v_{i k}-v_{i}\right), \\
& =\alpha_{j}\left(v_{i j}-v_{j}\right)+\beta_{j}\left(v_{j k}-v_{j}\right), \\
& =\alpha_{i j}\left(v_{j}-v_{i j}\right)+\beta_{i j}\left(u_{l}-v_{i j}\right) .
\end{aligned}
$$

For details on how to get the $\alpha$ 's and $\beta$ 's, see Remark 1 . We now use these equations to get formulae for $D_{n_{e}} s$ at certain points on $T$. First we note that for any two vertices $u, v$ of a triangulation $\triangle$, the directional derivative associated with the vector $u-v$ of a quadratic spline defined on $\triangle$ is

$$
D_{u-v} s(v)=2 \delta(u, v):=c(u, v)-c(v),
$$

see [4], p. 28. This leads to

$$
\begin{aligned}
D_{n_{e}} s\left(v_{i}\right) & =2\left[\alpha_{i} \delta\left(v_{i j}, v_{i}\right)+\beta_{i} \delta\left(v_{i k}, v_{i}\right)\right], \\
D_{n_{e}} s\left(v_{j}\right) & =2\left[\alpha_{j} \delta\left(v_{i j}, v_{j}\right)+\beta_{j} \delta\left(v_{j k}, v_{j}\right)\right], \\
D_{n_{e}} s\left(v_{i j}\right) & =2\left[\alpha_{i j} \delta\left(v_{j}, v_{i j}\right)+\beta_{i j} \delta\left(u_{l}, v_{i j}\right)\right] .
\end{aligned}
$$

The requirement that $D_{n_{e}} s$ be linear on $e$ can be written as

$$
D_{n_{e}} s\left(v_{i j}\right)=\left[D_{n_{e}} s\left(v_{i}\right)+D_{n_{e}} s\left(v_{j}\right)\right] / 2 .
$$

Now substituting the equations (3.5) and solving for $c\left(u_{l}, v_{i j}\right)$, we get

$$
\begin{aligned}
c\left(u_{l}, v_{i j}\right)=c\left(v_{i j}\right)+[ & -2 \alpha_{i j} \delta\left(v_{j}, v_{i j}\right)+\alpha_{i} \delta\left(v_{i j}, v_{i}\right)+\beta_{i} \delta\left(v_{i k}, v_{i}\right) \\
& \left.+\alpha_{j} \delta\left(v_{i j}, v_{j}\right)+\beta_{j} \delta\left(v_{j k}, v_{j}\right)\right] /\left(2 \beta_{i j}\right) .
\end{aligned}
$$




\section{$\S 4$. A Condensed $C^{1}$ Quadratic Tetrahedral Macro-Element}

In this section we apply the method of the previous section to construct a condensed version of the trivariate $C^{1}$ quadratic macro-element space described in [8]. Suppose $\triangle$ is a tetrahedral partition of a set $\Omega \subset \mathbb{R}^{3}$, and let $\mathcal{V}, \mathcal{E}, \mathcal{F}$ be the sets of vertices, edges, and faces, respectively. Let $\triangle_{M}$ be the associated partition obtained by applying the splitting process described in Algorithm 3.1 of [8] to each tetrahedron $T$ in $\triangle$. This refinement splits each $T$ into 504 subtetrahedra.

By the results of [8], the space $\mathcal{S}_{2}^{1}\left(\triangle_{M}\right)$ is a trivariate macro-element space whose dimension is $4 n_{V}+2 n_{E}+4 n_{F}$. For each vertex $v$ of $\triangle$, let

$$
\mathcal{N}_{v}:=\left\{\varepsilon_{v}, \varepsilon_{v} D_{x}, \varepsilon_{v} D_{y}, \varepsilon_{v} D_{z}\right\}
$$

where $\varepsilon_{v}$ denotes point-evaluation at $v$. For each face $F:=\left\langle v_{i}, v_{j}, v_{k}\right\rangle$ of $\triangle$, let $D_{F}$ be the derivative in a direction perpendicular to $F$, and let

$$
\mathcal{N}_{F}:=\left\{\varepsilon_{u_{l}} D_{F}, \varepsilon_{d_{l}^{i}} D_{F}, \varepsilon_{d_{l}^{j}} D_{F}, \varepsilon_{d_{l}^{k}} D_{F}\right\}
$$

where $u_{l}, d_{l}^{i}, d_{l}^{j}, d_{l}^{k}$ are four of the vertices of the PS-12 split of $F$ as shown in Fig. 1. For each edge $e$ of $\triangle$, let $e_{m}$ be its midpoint, and let $\mathcal{N}_{e}:=\left\{\varepsilon_{e_{m}} D_{e, 1}, \varepsilon_{e_{m}} D_{e, 2}\right\}$, where $D_{e, 1}$ and $D_{e, 2}$ are the derivatives corresponding to two directions $n_{e, 1}$ and $n_{e, 2}$ perpendicular to $e$. Then the results of [8] show that

$$
\mathcal{N}=\bigcup_{v \in \mathcal{V}} \mathcal{N}_{v} \cup \bigcup_{e \in \mathcal{E}} \mathcal{N}_{e} \cup \bigcup_{F \in \mathcal{F}} \mathcal{N}_{F}
$$

is a stable local NMDS for $\mathcal{S}_{2}^{1}\left(\triangle_{M}\right)$. Moreover, the corresponding dual $\mathcal{N}$-basis for $\mathcal{S}_{2}^{1}\left(\triangle_{M}\right)$ is a stable local basis. It follows from Theorem 17.22 of [4] that $\mathcal{S}_{2}^{1}\left(\triangle_{M}\right)$ approximates smooth functions to optimal order $\mathcal{O}\left(|\triangle|^{3}\right)$.

We now define a subspace of $\mathcal{S}_{2}^{1}\left(\triangle_{M}\right)$ which also is a macro-element space with full approximation power, but which requires nodal data only at the vertices of $\triangle$. It is clear that for every $s \in \mathcal{S}_{2}^{1}\left(\triangle_{M}\right)$ and every edge $e$ of $\triangle, D_{e, 1}$ and $D_{e, 2}$ are piecewise linear univariate functions on $e$. Similarly, for every face $F$ of $\triangle, D_{F} s$ is piecewise linear bivariate function on $F$. We define our condensed macro-element space by requiring these derivatives to be linear.

Definition 4.1. Given $\triangle_{M}$, we define the associated condensed $C^{1}$ quadratic trivariate macro-element space to be

$$
\begin{aligned}
\widetilde{\mathcal{S}}_{2}^{1}\left(\triangle_{M}\right):=\left\{s \in \mathcal{S}_{2}^{1}\left(\triangle_{M}\right):\right. & \left.D_{e, 1} s\right|_{e},\left.D_{e, 2} s\right|_{e} \in \mathcal{P}_{1}^{1} \text { for each edge } e \text { of } \triangle, \text { and } \\
& \left.\left.D_{F} s\right|_{F} \in \mathcal{P}_{1}^{2} \text { for each face } F \text { of } \triangle\right\} .
\end{aligned}
$$

Theorem 4.2. The dimension of $\mathcal{S}_{2}^{1}\left(\triangle_{M}\right)$ is $4 n_{V}$, and $\widetilde{\mathcal{N}}:=\bigcup_{v \in \mathcal{V}} \mathcal{N}_{v}$ is a stable local NMDS.

Proof: By the definition of $\widetilde{\mathcal{S}}_{2}^{1}\left(\triangle_{M}\right)$, if we set the values and gradients of $s \in$ $\widetilde{\mathcal{S}}_{2}^{1}\left(\triangle_{P S 12}\right)$ at the vertices of $\triangle$, then the cross derivatives $D_{e, 1}$ and $D_{e, 2}$ at the 
midpoints of the edges are uniquely determined by requiring $\left.D_{e} s\right|_{e}$ to be linear. But then the values of $D_{F}$ at the points $\left\{u_{l}, d_{l}^{i}, d_{l}^{j}, d_{l}^{k}\right\}$ associated with each face $F:=\left\langle v_{i}, v_{j}, v_{k}\right\rangle$ are also uniquely determined by requiring $\left.D_{F} s\right|_{F}$ to be linear, see the explicit formulae provided at the end of this section. The result then follows from the fact that $\mathcal{N}$ is a stable local NMDS for $\mathcal{S}_{2}^{1}\left(\triangle_{M}\right)$.

Corollary 4.3. For each $f \in C^{1}(\Omega)$, there exists a unique $s \in \widetilde{\mathcal{S}}_{2}^{1}\left(\triangle_{M}\right)$ such that

$$
s\left(v_{i}\right)=f\left(v_{i}\right) \quad \text { and } \quad \nabla s\left(v_{i}\right)=\nabla f\left(v_{i}\right), \quad i=1, \ldots, n .
$$

Moreover if $f \in \mathcal{P}_{2}^{3}$, then $s=f$.

Proof: The first statement is obvious due to the definition of $\widetilde{\mathcal{N}}$. Now suppose $f \in \mathcal{P}_{2}^{3}$. Then for each edge $e$ of $\triangle,\left.D_{e, 1} s\right|_{e}$ and $\left.D_{e, 2} s\right|_{e}$ are in $\mathcal{P}_{1}^{1}$, while for each face $F$ of $\triangle,\left.D_{F} s\right|_{F} \in \mathcal{P}_{1}^{2}$. Since $\mathcal{P}_{2}^{3} \subset \widetilde{\mathcal{S}}_{2}^{1}\left(\triangle_{M}\right)$ and there is a unique $s \in \widetilde{\mathcal{S}}_{2}^{1}\left(\triangle_{M}\right)$ satisfying (4.1), it follows that $s \equiv f$.

Since $\widetilde{\mathcal{N}}$ is stable and local, it follows from Theorem 17.22 of [4] that the Hermite interpolating spline defined in (4.1) approximates smooth functions to optimal order, and so the space $\widetilde{\mathcal{S}}_{2}^{1}\left(\triangle_{M}\right)$ has full approximation power.

As in the bivariate case we now provide explicit formula for computing the coefficients of a spline $s \in \widetilde{\mathcal{S}}_{2}^{1}\left(\triangle_{M}\right)$ satisfying (4.1). Let $T:=\left\langle v_{i}, v_{j}, v_{k}, v_{l}\right\rangle$ be a typical macro tetrahedron in $\triangle$. Then we can compute the coefficients of $s$ corresponding to domain points on the faces of $T$ from the nodal data in (4.1) by using the formulae at the end of Sect. 3. Let $w:=\left(v_{i}+v_{j}+v_{k}+v_{l}\right) / 4$ be the barycenter of $T$, and let $p_{i}:=\left(2 v_{i j}+v_{i k}+v_{i l}\right) / 3$ with similar definitions for $p_{j}$ and $p_{k}$ obtained by permuting the indices. We now give formulae for coefficients of the form $c\left(u_{l}, w\right)$ and $c\left(d_{l}^{i}, p_{i}\right)$. Once we have these, all remaining coefficients of $s$ can be computed from the explicit formulae in [8].

Let $F:=\left\langle v_{i}, v_{j}, v_{k}\right\rangle$ be a face of $T$. Then with the notation of Fig. 1, we can write

$$
\begin{aligned}
n_{F} & =\alpha_{i}\left(v_{i j}-v_{i}\right)+\beta_{i}\left(v_{i k}-v_{i}\right)+\gamma_{i}\left(v_{i l}-v_{i}\right), \\
& =\alpha_{j}\left(v_{i j}-v_{j}\right)+\beta_{j}\left(v_{j k}-v_{j}\right)+\gamma_{j}\left(v_{j l}-v_{j}\right), \\
& =\alpha_{k}\left(v_{i k}-v_{k}\right)+\beta_{k}\left(v_{j k}-v_{k}\right)+\gamma_{k}\left(v_{k l}-v_{k}\right), \\
& =\alpha_{l}\left(v_{i j}-u_{l}\right)+\beta_{l}\left(v_{i k}-u_{l}\right)+\gamma_{l}\left(w-u_{l}\right), \\
& =\alpha_{i l}\left(v_{i}-d_{l}^{i}\right)+\beta_{i l}\left(v_{i j}-d_{l}^{i}\right)+\gamma_{i l}\left(p_{i}-d_{l}^{i}\right) .
\end{aligned}
$$

For details on how to compute the $\alpha, \beta, \gamma$ 's, see Remark 2. Then using (3.4), we get the following formulae for the value of $D_{F} s:=D_{n_{F}} s$ at various points on $F$ :

$$
\begin{aligned}
& D_{F} s\left(v_{i}\right)=2\left[\alpha_{i} \delta\left(v_{i j}, v_{i}\right)+\beta_{i} \delta\left(v_{i k}, v_{i}\right)+\gamma_{i} \delta\left(v_{i l}, v_{i}\right)\right], \\
& D_{F} s\left(v_{j}\right)=2\left[\alpha_{j} \delta\left(v_{i j}, v_{j}\right)+\beta_{j} \delta\left(v_{j k}, v_{j}\right)+\gamma_{j} \delta\left(v_{j l}, v_{j}\right)\right], \\
& D_{F} s\left(v_{k}\right)=2\left[\alpha_{k} \delta\left(v_{i k}, v_{k}\right)+\beta_{k} \delta\left(v_{j k}, v_{k}\right)+\gamma_{k} \delta\left(v_{k l}, v_{k}\right)\right], \\
& D_{F} s\left(u_{l}\right)=2\left[\alpha_{l} \delta\left(v_{i j}, u_{l}\right)+\beta_{l} \delta\left(v_{i k}, u_{l}\right)+\gamma_{l} \delta\left(w, u_{l}\right)\right], \\
& D_{F} s\left(d_{l}^{i}\right)=2\left[\alpha_{i l} \delta\left(v_{i}, d_{l}^{i}\right)+\beta_{i l} \delta\left(v_{i j}, d_{l}^{i}\right)+\gamma_{i l} \delta\left(p_{i}, d_{l}^{i}\right)\right],
\end{aligned}
$$


where $\delta(u, v)$ is defined in (3.4). The requirement that $D_{F} s$ be linear on $F$ gives

$$
D_{F} s\left(u_{l}\right)=\left[D_{F} s\left(v_{i}\right)+D_{F} s\left(v_{j}\right)+D_{F} s\left(v_{k}\right)\right] / 3 \text {. }
$$

Now substituting the equations (4.3) and solving for $c\left(u_{l}, w\right)$, we get

$$
\begin{aligned}
c\left(u_{l}, w\right)=c\left(u_{l}\right)+[ & \alpha_{i} \delta\left(v_{i j}, v_{i}\right)+\beta_{i} \delta\left(v_{i k}, v_{i}\right)+\gamma_{i} \delta\left(v_{i l}, v_{i}\right)+\alpha_{j} \delta\left(v_{i j}, v_{j}\right) \\
& +\beta_{j} \delta\left(v_{j k}, v_{j}\right)+\gamma_{j} \delta\left(v_{j l}, v_{j}\right)+\alpha_{k} \delta\left(v_{i k}, v_{k}\right)+\beta_{k} \delta\left(v_{j k}, v_{k}\right) \\
& \left.+\gamma_{k} \delta\left(v_{k l}, v_{k}\right)-3 \alpha_{l} \delta\left(v_{i j}, u_{l}\right)-3 \beta_{l} \delta\left(v_{i k}, u_{l}\right)\right] /\left(3 \gamma_{l}\right) .
\end{aligned}
$$

Similarly, the requirement that $D_{F} s$ be linear on $F$ gives

$$
D_{F} s\left(d_{l}^{i}\right)=\left[2 D_{F} s\left(v_{i}\right)+D_{F} s\left(v_{j}\right)+D_{F} s\left(v_{k}\right)\right] / 4 \text {. }
$$

Substituting the equations (4.3) and solving for $c\left(d_{l}^{i}, p_{i}\right)$ gives

$$
\begin{aligned}
c\left(d_{l}^{i}, p_{i}\right)=c\left(d_{l}^{i}\right)+[ & 2 \alpha_{i} \delta\left(v_{i j}, v_{i}\right)+2 \beta_{i} \delta\left(v_{i k}, v_{i}\right)+2 \gamma_{i} \delta\left(v_{i l}, v_{i}\right)+\alpha_{j} \delta\left(v_{i j}, v_{j}\right) \\
& +\beta_{j} \delta\left(v_{j k}, v_{j}\right)+\gamma_{j} \delta\left(v_{j l}, v_{j}\right)+\alpha_{k} \delta\left(v_{i k}, v_{k}\right)+\beta_{k} \delta\left(v_{j k}, v_{k}\right) \\
& \left.+\gamma_{k} \delta\left(v_{k l}, v_{k}\right)-4 \alpha_{i l} \delta\left(v_{i}, d_{l}^{i}\right)-4 \beta_{i l} \delta\left(v_{i j}, d_{l}^{i}\right)\right] /\left(4 \gamma_{i l}\right) .
\end{aligned}
$$

\section{$\S 5$. The Effect of Condensation}

In this section we give examples in both the bivariate and trivariate cases to show that our condensed elements have the correct orders of approximation, and that on some test examples, the errors for our methods are not much worse than those for the uncondensed elements.

To test our condensed PS-12 element, we use the well-known Franke function $f$ (cf., [3]) on the unit square $\Omega$. To conduct the test we constructed a nested sequence of type-I triangulations with 25, 49, 81, 289, and 1089 vertices. Table 1 shows the results of interpolating $f$ from the spaces $\mathcal{S}_{2}^{1}\left(\triangle_{P S 12}\right)$ and $\widetilde{\mathcal{S}}_{2}^{1}\left(\triangle_{P S 12}\right)$. The columns labeled $e_{\infty}$ and $\tilde{e}_{\infty}$ give the maximum errors on a grid of 25,000 points in $\Omega$, while those labeled $e_{2}$ and $\tilde{e}_{2}$ give the RMS errors on the same grid.

There seems to be no generally recognized standard test function for trivariate problems. To test our condensed trivariate element, we work with the cubic test function $f(x, y, z)=x^{3}+y^{3}+z^{3}$ on the unit cube. The error expressions for the tetrahedral scheme involve third order derivatives of the test functions. These are constant for a cubic function, and higher order derivatives are zero. Thus, the error behavior should be close to the theoretic expectations. To conduct the test we constructed a nested sequence of Delaunay (cf., [5]) tetrahedral partitions with 8, $27,125,729$, and 2197 vertices on an equally-spaced grid. Table 2 shows the results of interpolating $f$ from the spaces $\mathcal{S}_{2}^{1}\left(\triangle_{M}\right)$ and $\widetilde{\mathcal{S}}_{2}^{1}\left(\triangle_{M}\right)$. The columns labeled $e_{\infty}$ 


\begin{tabular}{|c|c|c|c|c|}
\hline$n$ & $e_{\infty}$ & $e_{2}$ & $\tilde{e}_{\infty}$ & $\tilde{e}_{2}$ \\
\hline 25 & $7.55(-2)$ & $1.50(-2)$ & $8.03(-2)$ & $1.58(-2)$ \\
49 & $5.36(-2)$ & $5.72(-3)$ & $5.94(-2)$ & $6.39(-3)$ \\
81 & $1.91(-2)$ & $1.91(-3)$ & $2.02(-2)$ & $2.17(-3)$ \\
289 & $2.13(-3)$ & $1.66(-4)$ & $2.27(-3)$ & $1.98(-4)$ \\
1089 & $1.85(-4)$ & $1.57(-5)$ & $1.88(-4)$ & $1.94(-5)$ \\
\hline
\end{tabular}

Tab. 1. Errors for $\mathcal{S}_{2}^{1}\left(\triangle_{P S 12}\right)$ and $\widetilde{\mathcal{S}}_{2}^{1}\left(\triangle_{P S 12}\right)$.

\begin{tabular}{|c|c|c|c|c|}
\hline$n$ & $e_{\infty}$ & $e_{2}$ & $\tilde{e}_{\infty}$ & $\tilde{e}_{2}$ \\
\hline 8 & $5.01(-2)$ & $1.60(-2)$ & $9.29(-2)$ & $3.65(-2)$ \\
27 & $6.21(-3)$ & $1.83(-3)$ & $1.72(-2)$ & $6.75(-3)$ \\
125 & $7.77(-4)$ & $2.26(-4)$ & $2.13(-3)$ & $8.56(-4)$ \\
729 & $9.48(-5)$ & $2.80(-5)$ & $2.63(-4)$ & $1.06(-4)$ \\
2197 & $2.71(-5)$ & $8.20(-6)$ & $7.50(-5)$ & $3.07(-5)$ \\
\hline
\end{tabular}

Tab. 2. Errors for $\mathcal{S}_{2}^{1}\left(\triangle_{M}\right)$ and $\widetilde{\mathcal{S}}_{2}^{1}\left(\triangle_{M}\right)$.

and $\tilde{e}_{\infty}$ give the maximum errors on a grid of one million points in $\Omega$, while those labeled $e_{2}$ and $\tilde{e}_{2}$ give the RMS errors on the same grid.

Since condensation of parameters does not change the approximation power of the macro-element schemes, it should only affect the constants in the error expansion. The errors in the condensed and uncondensed schemes should therefore be roughly proportional. This is borne out by the numerical results, with the constant of proportionality being approximately 1 in the bivariate case and approximately 3 in the trivariate case.

The expected rate of convergence for our schemes is $n^{-1.5}$ for the bivariate scheme, and $n^{-1}$ for the trivariate scheme. This is roughly consistent with the numerical results. We also verified numerically that both of our schemes reproduce quadratic polynomials exactly.

\section{$\S 6$. Remarks}

Remark 1. To find $\alpha_{i}$ and $\beta_{i}$ in the first equation of (3.3), we first compute $a$ so that $\left[a\left(v_{i j}-v_{i}\right)+\left(v_{i k}-v_{i}\right)\right] \circ\left(v_{i j}-v_{i}\right)=0$. This is one equation for the unknown $a$. Then we normalize $a\left(v_{i j}-v_{i}\right)+\left(v_{i k}-v_{i}\right)$ to get $n_{e}$.

Remark 2. To find $\alpha_{i}, \beta_{i}, \gamma_{i}$ in the first equation of (4.2), we first compute $a, b$ so that $a\left(v_{i j}-v_{i}\right)+b\left(v_{i k}-v_{i}\right)+\left(v_{i l}-v_{i}\right)$ is orthogonal to $v_{i j}-v_{i}$ and $v_{i k}-v_{i}$. This gives two equations in the two unknowns $a$ and $b$. Then we normalize $a\left(v_{i j}-v_{i}\right)+$ $b\left(v_{i k}-v_{i}\right)+\left(v_{i l}-v_{i}\right)$ to get $n_{F}$.

Remark 3. In defining our condensed version of the PS-12 macro-element, we have made perpendicular cross-boundary derivatives be linear instead of piecewise linear. This does not imply that cross derivatives in other directions are linear. 
They are linear combinations of the linear perpendicular cross boundary derivatives and certain other derivatives that are only piecewise linear.

Remark 4. In defining our condensed trivariate scheme, for each edge $e$ we have made the derivatives corresponding to two particular directions perpendicular to $e$ be linear. Since all directions perpendicular to an edge are combinations of these two, it follows that the derivative of $s$ in any direction perpendicular to $e$ is linear on $e$. Thus, our scheme is independent of the specific choice of perpendicular directions to be made linear.

Remark 5. The approach to condensation of parameters described in this paper depends heavily on the fact that the derivatives of the macro-elements restricted to edges (and/or faces in the trivariate case) are piecewise polynomials rather than single polynomials. Many of the classical macro-elements do not have this property, and thus are not amenable to the approach described here.

Remark 6. Another approach to condensation, as described for example in [1], is to make cross derivatives along edges or faces of a partition have lower degree than they normally would have. This approach applies to most macro-element spaces, but has the disadvantage that the resulting condensed spaces no longer have full approximation power.

Remark 7. As shown in [7], the macro-element space $\mathcal{S}_{2}^{1}\left(\triangle_{P S 12}\right)$ is just one member of a family of $C^{r}$ macro-element spaces defined on PS-12 refinements of triangulations $\triangle$. We believe that these spaces can be condensed using the methods of this paper, but not with the NMDS's given in [7]. We will treat this problem in a separate paper.

\section{References}

1. Alfeld, P., A trivariate Clough-Tocher scheme for tetrahedral data, Comput. Aided Geom. Design 1 (1984), 169-181.

2. Alfeld, P. and T. Sorokina, Two Tetrahedral $C^{1}$ Cubic Macro Elements, J. Approx. Theory, DOI: 10.1016/j.jat.2008.07.001, to appear.

3. Franke, Richard, Scattered data interpolation: tests of some methods, Math. Comp. 38(157) (1982), 181-200.

4. Lai, M. J. and L. L. Schumaker, Spline Functions on Triangulations, Cambridge University Press, Cambridge, 2007.

5. Lawson, C. L., Properties of $n$-dimensional triangulations, Comput. Aided Geom. Design 3 (1986), 231-247.

6. Powell, M. J. D. and M. A. Sabin, Piecewise quadratic approximations on triangles, ACM Trans. Math. Software 3 (1977), 316-325.

7. Schumaker, L. L. and T. Sorokina, A family of $C^{r}$ Macro-elements on PowellSabin-12 splits, Math. Comp. 75 (2006), 711-726. 
8. Schumaker, L. L., T. Sorokina, and A. J. Worsey, A $C^{1}$ Quadratic trivariate macro-element space defined over arbitrary tetrahedral partitions, J. Approx. Theory, DOI: 10.1016/j.jat.2008.04.014, to appear. 